\title{
ADSORÇÃO DE FENOL NO CARVÃO ATIVADO PRODUZIDO A PARTIR DO ENDOCARPO DO FRUTO DA MACAÚBA
}

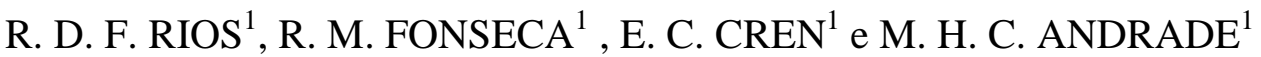 \\ ${ }^{1}$ Universidade Federal de Minas Gerais, Departamento de Engenharia Química \\ E-mail para contato: regiane.debora@yahoo.com.br
}

\begin{abstract}
RESUMO - A palmeira Macaúba (Acrocomia aculeata) produz um fruto oleaginoso contendo um endocarpo, cerca de $25 \%$ em massa, teor de lignina $(40 \%)$ e elevada resistência mecânica, com potencial para a produção de carvão ativado. Neste trabalho, carvão ativado, foi utilizado em ensaios de adsorção nas temperaturas de 30,40 e $50^{\circ} \mathrm{C}$, para concentrações de solução de fenol de 200 e 40 ppm, simulando duas etapas de adsorção em série. Os resultados indicaram ser possível obter solução final com $5 \mathrm{mg} / \mathrm{l}$ de fenol. Em todas as temperaturas, o modelo de isoterma de Langmuir melhor se ajustou aos dados, indicando que o carvão apresenta sítios idênticos entre si, com uma molécula adsorvida por sítio e sem interações relevantes entre as mesmas. O fenômeno da adsorção de fenol foi adequadamente representado pelos modelos cinéticos de pseudo-primeira ordem e pseudo-segunda ordem, sendo a adsorção química indicada como a etapa limitante do processo.
\end{abstract}

\section{INTRODUÇÃO}

O fenol, efluente comum em diversas indústrias, é altamente tóxico e cancerígeno. Assim, é importante que esses efluentes sejam tratados antes de serem liberados no meio-ambiente. O composto é um resíduo muito comum em coquearias, plantas petroquímicas e refinarias. Nesse último, o craqueamento catalítico em leito fluidizado gera efluentes contendo concentrações consideráveis de fenol (50-260 ppm massa) Britto (2008). A concentração máxima de fenol permitidas pela resolução CONAMA como padrão de lançamento para efluentes industriais é de 0,5 $\mathrm{mg} / \mathrm{L}$ de fenóis totais. Uma forma para retirada do fenol desses efluentes é a adsorção do mesmo em sistemas com carvão ativado, os quais apresentam alta habilidade de adsorção de moléculas de baixo peso molecular.

A Macaúba (Acrocomia aculeata) é uma palmeira nativa das florestas tropicais, originária das matas do norte ao sudeste do Brasil e constituído de um epicarpo duro (casca externa), de um mesocarpo fibroso e oleoso (polpa), de uma ou duas amêndoas oleosas na região mais interna e de um endocarpo duro que representa cerca de $25 \%$ de sua massa e cujas características principais são alto teor de lignina (cerca de 40\%) e elevada resistência mecânica, características que viabilizam o seu uso para a produção de um carvão vegetal economicamente competitivo, sem promover degradação da natureza. 
Neste trabalho, busca-se reduzir o teor de fenol de soluções com concentrações iniciais similares às dos efluentes industriais, a fim de atingir concentrações próximas àquelas determinadas pela legislação ambiental vigente. Para isso, utilizou-se a técnica de adsorção de fenol em carvão ativado produzido a partir do endocarpo do fruto da Macaúba, produzido pelo Grupo de Pesquisa Processos e Produtos da Macaúba, do Departamento de Engenharia Química da UFMG.

\section{PARTE EXPERIMENTAL}

\subsection{Caracterização Do Carvão}

O carvão ativado a partir do endocarpo da Macaúba utilizado no estudo foi produzido e sua caracterização foi previamente realizada. Para a composição química utilizou-se a técnica de gravimetria, norma ABNT NBR-8112; o teor de umidade foi determinado por gravimetria, norma ABNT-NBR 8293; a densidade foi quantificada pelo método direto de determinação da massa e volume aparente da amostra; $\mathrm{pH}$ pela norma norma JIS (Japanese Industrial Standard)a umidade) e; a quantificação da área superficial, volume e tamanho de poro foi obtida por análise BET, conduzida em um porosímetro automático da marca QUANTA CHROME, modelo NOVA 1000, conectado a um microcomputador. A Tabela 1 a seguir apresenta os dados de caracterização física e composição do carvão ativado.

Tabela 1- Composição e propriedades do carvão ativado

\begin{tabular}{|c|c|}
\hline Materiais voláteis (\%) & 20 \\
\hline Cinzas (\%) & 10 \\
\hline Carbono Fixo (\%) & 70 \\
\hline Densidade $(\mathrm{g} / \mathrm{mL})$ & 0,89 \\
\hline Teor de Umidade (\%) & 1 \\
\hline $\mathrm{pH}$ & 7 \\
\hline Área Superficial $\left(\mathrm{m}^{2} / \mathrm{g}\right)$ & 286,10 \\
\hline Volume de poro $\left(\mathrm{cm}^{3} / \mathrm{g}\right)$ & 0,158 \\
\hline Tamanho de Poro $(\mathrm{nm})$ & 1,948 \\
\hline
\end{tabular}

\subsection{Teste Cinético}

Testes cinéticos foram realizados em duplicata utilizando $2 \mathrm{~g}$ do carvão ativado produzido em $250 \mathrm{~mL}$ de solução inicial de fenol em duas concentrações, $200 \mathrm{mg} / \mathrm{L}$ e 40mg/L. Os ensaios foram realizados em erlenmeyers dispostos em banho termostático agitado a $160 \mathrm{rpm}$, mantido a $30^{\circ} \mathrm{C}$. Alíquotas com aproximadamente $4 \mathrm{~mL}$ de cada amostra foram retiradas em intervalos de 20 minutos (alíquotas 1 a 3), 30 minutos (alíquotas 4 a 16) e 24 horas (alíquota 17) e analisadas em espectrofotômetro UV-Vis, marca Perkin Elmer Lambda XLS, em comprimento de onda de $650 \mathrm{~nm}$. 
Os resultados experimentais dos testes cinéticos foram utilizados para avaliar a adequação dos seguintes modelos de literatura: pseudo-primeira ordem, pseudo segunda ordem, difusão intrapartícula e Bangnhan (Schneider, 2008).

\subsection{Teste Termodinâmico}

Testes termodinâmicos foram realizados em duplicata, para diferentes massas iniciais de carvão ativado $(1,2,3$ e $4 \mathrm{~g})$ e temperaturas $\left(30^{\circ} \mathrm{C}, 40^{\circ} \mathrm{C}\right.$ e $\left.50^{\circ} \mathrm{C}\right)$. Os ensaios foram realizados em erlenmeyers contendo $200 \mathrm{~mL}$ de solução inicial de fenol de $200 \mathrm{mg} / \mathrm{L}$, dispostas no banho termostático nas temperaturas indicadas e agitação de $160 \mathrm{rpm}$. Após 24 horas, alíquotas das amostra foram retiradas e analisadas em espectrofotômetro UV-Vis, em comprimento de onda de $650 \mathrm{~nm}$.

Os resultados foram ajustados pelos modelos de Langmuir (1918), Freundlich (Sposito, 1980) e Temkin (Schneider, 2008), descritos na Tabela 2.

Tabela 2 - Modelos de Isotermas

\begin{tabular}{|c|c|c|c|}
\hline Isoterma & Característica & Equação & Equação Linearizada \\
\hline Langmuir & $\begin{array}{c}\text { O modelo foi o primeiro } \\
\text { a supor que ocorre a } \\
\text { formação de uma } \\
\text { monocamada na } \\
\text { superfície do } \\
\text { adsorvente. }\end{array}$ & $q_{e}=\frac{k_{L^{*}} q_{\max } C_{e}}{1+k_{L^{*}} C_{e}}$ & $\frac{C_{e}}{q_{e}}=\frac{1}{k_{L^{*}} q_{\max }}+\frac{C_{e}}{q_{\max }}$ \\
\hline Freundlich & $\begin{array}{c}\text { Apresenta bons } \\
\text { resultados para } \\
\text { adsorção sobre } \\
\text { superfícies } \\
\text { heterogêneas, como é o } \\
\text { caso do carvão ativado. }\end{array}$ & $q_{e}=k_{F^{*}} C_{e}{ }^{1 / n}$ & $\log \left(q_{\theta}\right)=\log \left(k_{F}\right)+\left(\frac{1}{n}\right) \cdot \log \left(C_{e}\right)$ \\
\hline Temkin & $\begin{array}{c}\text { Contém um fator capaz } \\
\text { de descrever as } \\
\text { interações adsorbato- } \\
\text { adsorvente }\end{array}$ & $q_{\theta}=\frac{R \cdot T}{b} \ln \left(k_{T^{*}} \cdot C_{e}\right)$ & \\
\hline
\end{tabular}

onde $\mathrm{q}_{\max }$ é a capacidade de adsorção máxima (mg. $\left.\mathrm{g}^{-1}\right), \mathrm{q}_{\mathrm{e}}$ a quantidade do adsorbato adsorvida no equilíbrio (mg.g $\left.{ }^{-1}\right), C_{e}$ a concentração do adsorbato no equilíbrio $\left(\mathrm{mg} . \mathrm{L}^{-1}\right), \mathrm{k}_{\mathrm{L}}$ constante equivalente à constante de equilíbrio químico em reações (L.mg-1), $\mathrm{k}_{\mathrm{T}}$ a constante de equilíbrio de ligação $\left(\mathrm{L}_{\mathrm{mg}} \mathrm{m}^{-1}\right), \mathrm{k}_{\mathrm{F}}\left[\left(\mathrm{mg} \cdot \mathrm{g}^{-1}\right) .\left(\mathrm{L} \cdot \mathrm{mg}^{-1}\right)^{1 / \mathrm{n}}\right]$ a constante que representa a capacidade de adsorção, $\mathrm{b} o$ calor de adsorção no início do processo $\left(\mathrm{kJ}^{\mathrm{m}} \mathrm{mol}^{-1}\right), \mathrm{R}$ a constante universal dos gases $\left(8,314 \mathrm{~J} \mathrm{~K}^{-1} \mathrm{~mol}^{-}\right.$ ${ }^{1}$ ) ,n uma constante adimensional que representa a intensidade do processo de adsorção e $\mathrm{T}$ a 
temperatura $(\mathrm{K})$.

\section{RESULTADOS E DISCUSSÕES}

\subsection{Teste Cinético}

Nas Figuras 1 e 2, pode-se observar que a concentração de fenol decai com o tempo, e como a quantidade acumulada adsorvida cresce. Na figura 1, como era de se esperar, ocorre uma queda acentuada na concentração de fenol no início do processo nas duas concentrações, sendo a solução mais concentrada no início, a adsorção ocorre mais rapidamente, com a velocidade decrescendo à medida que a solução fica menos concentrada. Após as medidas iniciais, o experimento foi deixado durante o período noturno, esperando-se o tempo de 24 horas para a tomada do último ponto, o qual foi admitido como o ponto de equilíbrio entre os fenômenos de adsorção e dessorção, conforme tendência de estabilização das quantidades mensuradas de concentração final e quantidade adsorvida. O resultado apresentado para o ensaio com concentração inicial de $200 \mathrm{mgL}^{-1}$ considera a média aritmética das absorbâncias medidas com o respectivo desvio padrão dos ensaios feitos em duplicata, sendo que a concentração final de fenol no equilíbrio foi de $16,11+/-2,59 \mathrm{mgL}^{-1}$. Os ensaios com concentração inicial de $40 \mathrm{mgL}^{-1}$ também ocorreram em duplicata, contudo, para um dos ensaios, ao final do tempo de 24 horas foi constatado um aumento do quantitativo de fenol na solução, indicativo do fenômeno da dessorção, o que explica o elevado desvio padrão observado na média dos valores de concentração final de equilíbrio, igual a $11,53+/-8,95 \mathrm{mgL}^{-1}$, enquanto que para um dos ensaios o valor da concentração final foi de $5,2 \mathrm{mgL}^{-1}$. Este resultado está sendo objeto de nova investigação.

No gráfico da

Figura 2, o último ponto representa a quantidade máxima de fenol adsorvida por grama de carvão, aproximadamente 22,98 mg de fenol / g de carvão e 3,56 mg de fenol/ g de carvão, respectivamente. Observa-se que a diferença dos valores era esperado uma vez que uma das forças motrizes do processo (concentração de fenol) foram alteradas em larga escala, modificando a concentração de equilíbrio.

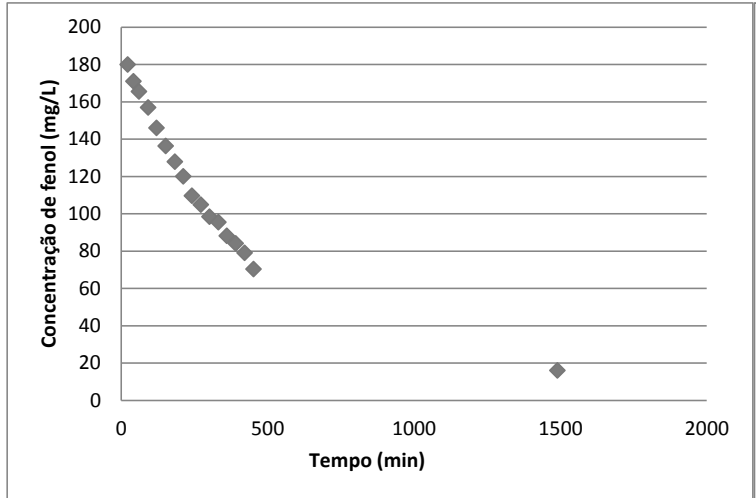

(a)

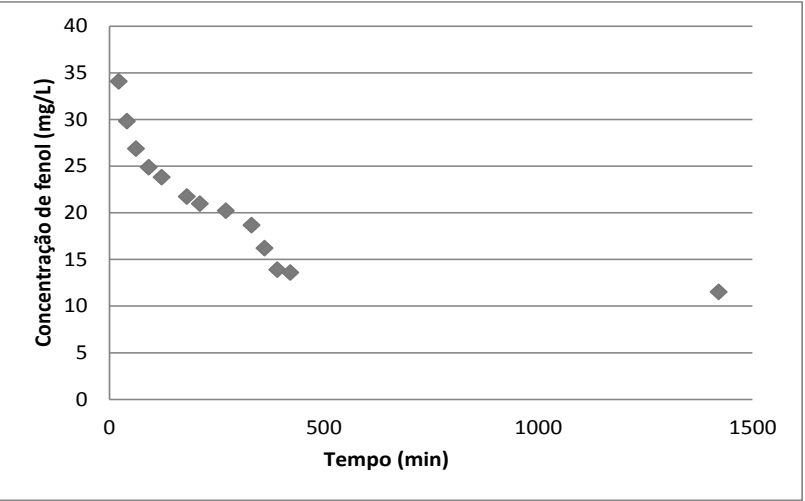

(b)

Figura 1- Curva cinética para solução de $200 \mathrm{mg} / \mathrm{L}$ (a) e $40 \mathrm{mg} / \mathrm{L}$ (b). 


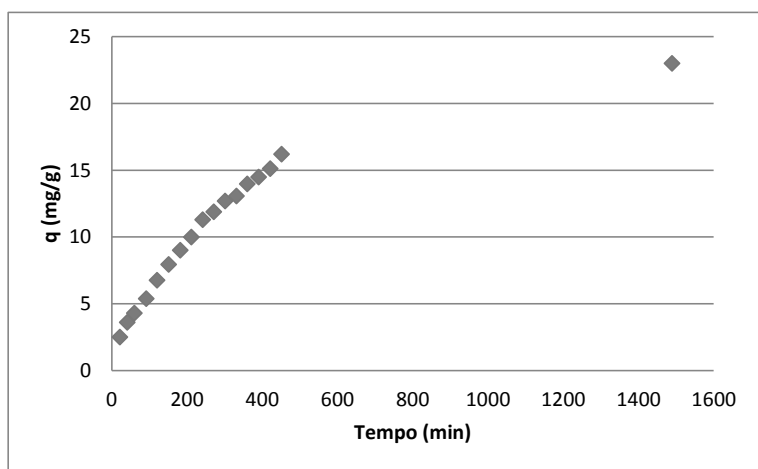

(a)

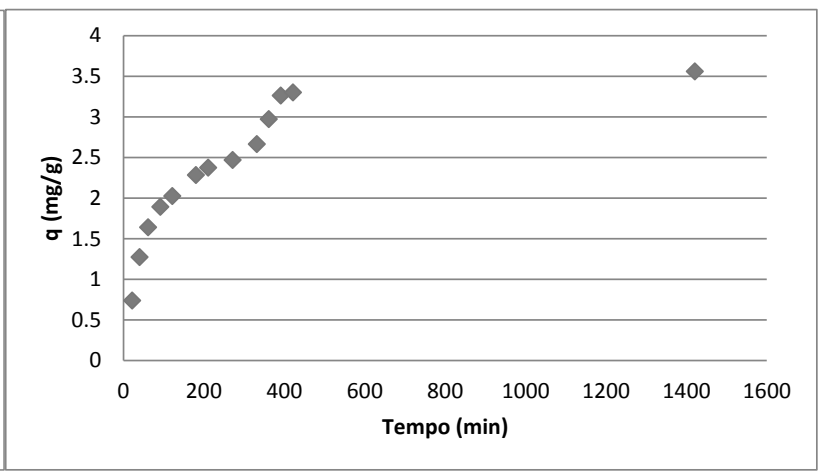

(b)

Figura 2- Quantidade de fenol adsorvida acumulada por grama de carvão com o tempo para a solução de $200 \mathrm{mg} / \mathrm{L}$ (a esquerda) e $40 \mathrm{mg} / \mathrm{L}$ (a direita).

Como forma de melhor tomar uma decisão sobre o modelo que melhor representa a cinética de adsorção estudada, a partir do ajuste dos dados experimentais dos modelos de literatura, foram comparados os coeficientes de correlação linear, sendo os resultados apresentados na Tabela 3.

Tabela 3- $\mathrm{R}^{2}$ dos modelos para todos os testes realizados.

\begin{tabular}{|c|c|c|c|c|}
\hline $\begin{array}{c}\text { Modelo / } \\
\text { Experimento }\end{array}$ & $\begin{array}{c}\text { Pseudo-primeira } \\
\text { ordem }\end{array}$ & $\begin{array}{c}\text { Pseudo-segunda } \\
\text { ordem }\end{array}$ & $\begin{array}{c}\text { Difusão } \\
\text { intrapartícula }\end{array}$ & Bangham \\
\hline $\begin{array}{c}2 \mathrm{~g} \text { carvão, } \\
(200 \mathrm{mg} / \mathrm{L})\end{array}$ & $\mathbf{0 , 9 9 6 6}$ & 0,9828 & 0,9487 & 0,9920 \\
\hline $\begin{array}{c}2 \mathrm{~g} \text { carvão, } \\
(40 \mathrm{mg} / \mathrm{L})\end{array}$ & 0,8957 & $\mathbf{0 , 9 9 4 3}$ & 0,7906 & 0,9349 \\
\hline
\end{tabular}

Conforme destacado na Tabela 3, o modelo de pseudo-primeira ordem foi o que apresentou melhor coeficiente para o caso da solução com concentração inicial maior, enquanto que o modelo de pseudo-segunda ordem representou melhor o comportamento da adsorção para solução de menor concentração. Ainda, verifica-se que o modelo de difusão intrapartícula representou os dados de forma muito inferior aos outros três modelos. Como este modelo supõe que a etapa de difusão intrapartícula é a limitante do processo, pode-se concluir que esta não é a etapa limitante do processo.

De um modo geral, os gráficos cinéticos podem apresentar multilinearidade, supondo que duas ou mais etapas podem ser controladoras da velocidade de adsorção em instantes diferentes do processo. Uma explicação para esta multilinearidade é o fato das constantes dos modelos variarem com o tempo, ou até mesmo com a concentração de sítios livres na fase sólida (HO et. al, 1996). Os resultados indicam a adsorção química como a etapa determinante para a velocidade do processo. 


\subsection{Testes Termodinâmicos}

Modelos de Isoterma: Os testes de equilíbrio termodinâmico foram realizados com 4 massas distintas de carvão para as temperaturas de 30,40 e $50^{\circ} \mathrm{C}$. Os resultados obtidos foram ajustados para os modelos de Langmuir, Freundlich e Temkin, sendo os resultados de $\mathrm{R}^{2}$ para cada modelo em cada temperatura analisada apresentados na Tabela 4.

Tabela 4 - $\mathrm{R}^{2}$ encontrado na linearização dos modelos de isotermas estudados

\begin{tabular}{|c|c|c|c|}
\hline $\begin{array}{c}\text { Modelos/ } \\
\mathrm{T}\left({ }^{\circ} \mathrm{C}\right)\end{array}$ & Langmuir & Freundlich & Temkin \\
\hline 30 & 0,9991 & 0,9634 & 0,9934 \\
\hline 40 & 0,9988 & 0,8726 & 0,9029 \\
\hline 50 & 0,9296 & 0,9047 & 0,8100 \\
\hline
\end{tabular}

Conforme Tabela 4, o modelo de Langmuir propicia um melhor ajuste dos dados em relação aos outros modelos (Langmuir, 1918). Esse resultado indica que o carvão utilizado apresenta sítios idênticos entre si, que possuem mesma energia de ativação durante todo o processo de adsorção, com apenas uma molécula adsorvida por sítio e que não há interações relevantes entre as moléculas de fenol adsorvidas. Adicionalmente, indica a formação de uma monocamada, condição estabelecida no modelo de Langmuir, que é típica do fenômeno de quimiossorção, resultado igualmente obtido pelos testes cinéticos parametrizados pelo modelo de pseudo-segunda ordem. Conclusões semelhantes para a adsorção de fenol com ajuste mais adequado ao modelo de Langmuir foram obtidas por Rodrigues (2011) e Schneider (2008).

Outro aspecto a ser ressaltado em relação aos resultados é o pior ajuste dos dados da temperatura de $50^{\circ} \mathrm{C}$ para os modelos considerados. Valores de $\mathrm{R}^{2}$ obtidos tanto por este trabalho quanto por diversos outros apresentados na Revisão Bibliográfica são considerados bons quando acima de 0.9900 . Se comparado a esse valor de referência, o $\mathrm{R}^{2}=0.9296$ obtido para o modelo de Langmuir não é suficiente para afirmar que o fenômeno de adsorção segue realmente esse modelo a essa temperatura.

Comparativo entre 30,40 e $50^{\circ} \mathrm{C}$ : Uma vez que o modelo de Langmuir foi encontrado como o que melhor representa o sistema, foi utilizada a Equação linearizada de Langmuir para o cálculo dos parâmetros $\mathrm{q}_{\max }$ e $\mathrm{k}_{\mathrm{L}}$ da Isoterma de Langmuir. Assim, a Tabela 5 apresenta, para as diferentes temperaturas avaliadas, os parâmetros de Langmuir e os valores médios para a concentração no equilíbrio, $\mathrm{C}_{\mathrm{e}}$, para as diferentes massas de carvão utilizadas no experimento. Ressalta-se que os testes termodinâmicos partiram da concentração de $200 \mathrm{mg} / \mathrm{L}$ de fenol. As isotermas para cada temperatura são mostradas na Erro! Fonte de referência não encontrada.3.

Tabela 5- Parâmetros da Equação da Isoterma de Langmuir e Concentrações de Equilíbrio.

\begin{tabular}{|c|c|c|c|}
\hline $\begin{array}{c}\mathrm{T}\left({ }^{\circ} \mathrm{C}\right) / \\
\text { Parâmetros }\end{array}$ & 30 & 40 & 50 \\
\hline $\mathrm{q}_{\max }(\mathrm{mg} / \mathrm{g})$ & 21,98 & 17,01 & 25,71 \\
\hline $\mathrm{K}_{\mathrm{L}}(\mathrm{L} / \mathrm{mg})$ & 0,19 & 0,17 & 0,11 \\
\hline
\end{tabular}



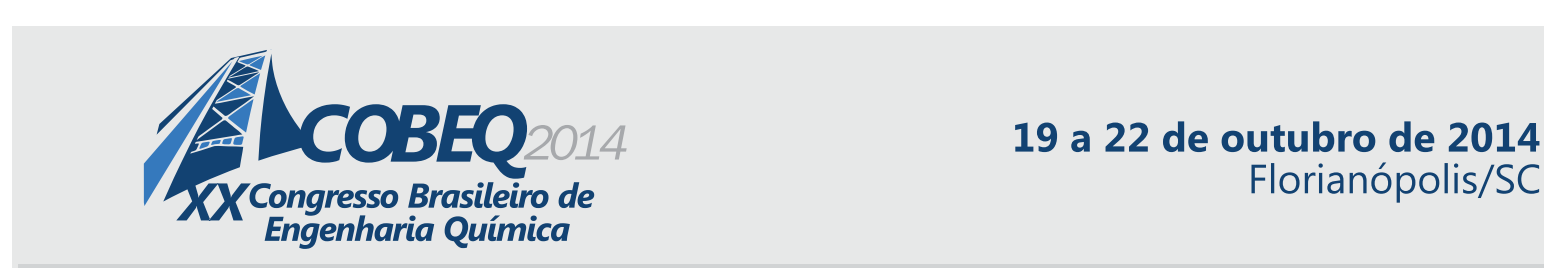

Florianópolis/SC

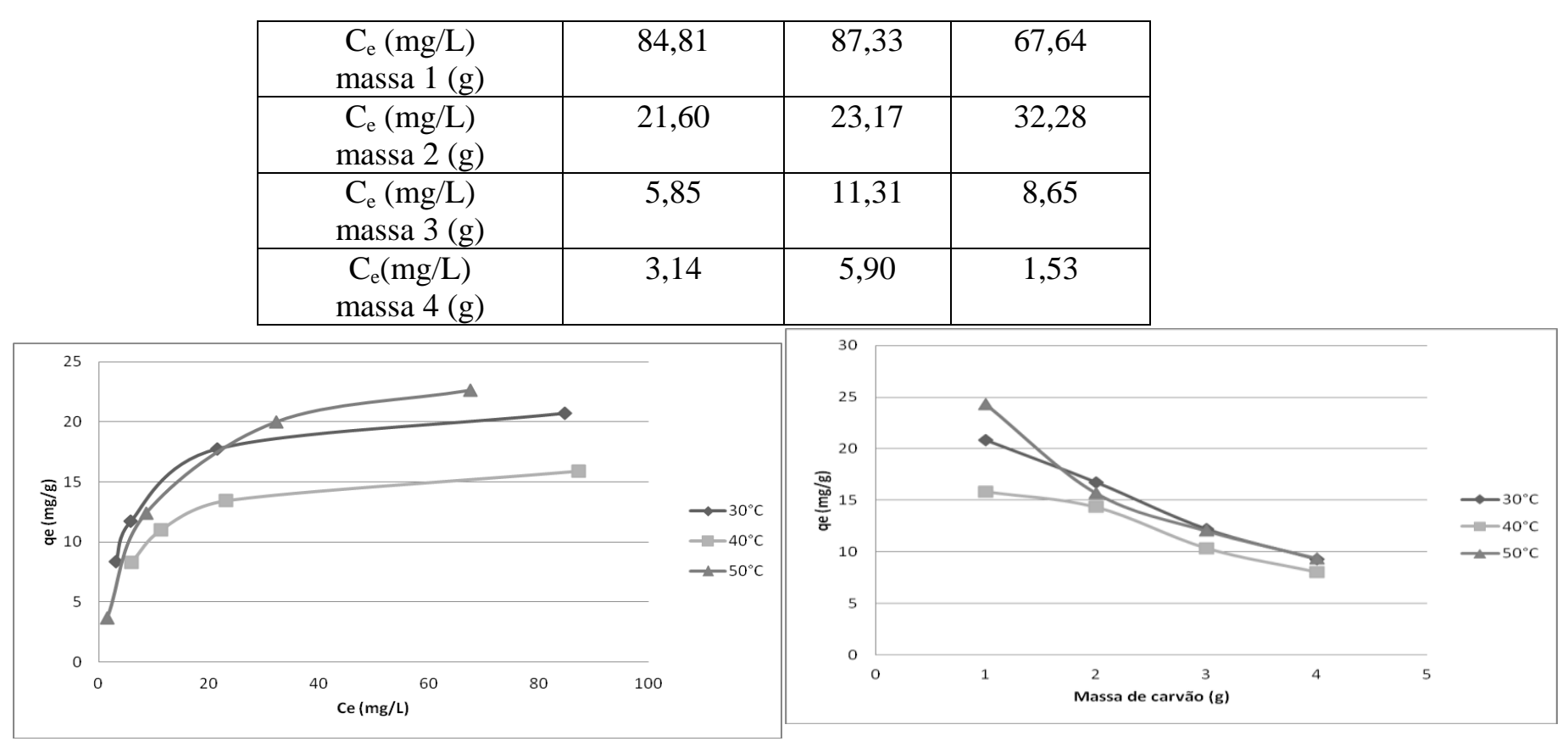

Figura 3- Isotermas de Langmuir a 30, 40 e $50^{\circ} \mathrm{C}$ para a adsorção do fenol.( a esquerda) e efeito do aumento da massa de carvão ativado na eficiência de adsorção (a direita).

Sabe-se que a adsorção é um processo exotérmico, logo um aumento de temperatura desfavorece esse processo. Conforme Figura 3, esse comportamento foi observado na comparação entre as temperaturas de 30 e $40^{\circ} \mathrm{C}$ : a eficiência de adsorção no equilíbrio $\left(\mathrm{q}_{\mathrm{e}}\right)$ é maior para temperaturas menores.

No entanto, para a temperatura de $50{ }^{\circ} \mathrm{C}$ foi observada uma maior adsorção e situações não identificadas no experimento podem ser a causa, principalmente destacando que o mecanismo a $50^{\circ} \mathrm{C}$ não pode ser expresso pelo modelo de Langmuir $\left(\mathrm{R}^{2}=0,9296\right)$ e, portanto, os resultados não são muito confiáveis, sendo que o aumento da temperatura pode ter interferido na etapa controladora do processo. Cabe ressaltar que o modelo mal ajustado é capaz de explicar o elevado $\mathrm{q}_{\max }$ para a temperatura de $50^{\circ} \mathrm{C}$, mas não explica a efetiva redução da concentração de fenol observada experimentalmente.

Conforme Marsh \& Reinoso(2006), uma outra possiblidade é a reação do fenol com algum grupo funcional do carvão. Dentre esses grupos possíveis, o fenol pode apresentar reação com o anidrido carboxílico e com o ácido carboxílico (embora a reação seja lenta) formando ésteres. Embora não tenha sido feita a análise de grupos funcionais presentes no carvão, essa hipótese deve ser considerada uma vez que os resultados da Tabela 5 indicam que para uma maior quantidade de carvão, maior foi a eficiência de retirada do fenol a $50^{\circ} \mathrm{C}$. Ou seja, maiores valores massa, portanto maior disponibiilidade de grupos funcionais, e maior temperatura, maior será o favorecimento da reação do fenol com algum grupo funcional do carvão.

Em síntese, as situações não identificadas podem ser do tipo: (i) erros na condução experimental; (ii) não adequação do modelo de Langmuir e: (iii) possibilidade de reação do fenol com grupo funcional do carvão. Essa situações devem ser melhor averiguadas. 


\section{CONCLUSÃO}

Neste trabalho foi testada a efetividade do carvão ativado obtido do endocarpo do fruto da Macaúba na adsorção de fenol em soluções aquosas.

Para os testes realizados partindo de uma solução de $40 \mathrm{mg} / \mathrm{L}$ e utilizando carvão ativado, chegou-se a uma concentração final de fenol de aproximadamente 5,2 mg/L. Essa concentração ainda é superior às normas legislativas brasileiras, as quais estabelecem concentração de fenol menor que $0,5 \mathrm{mg} / \mathrm{L}$ em efluentes.

O estudo da cinética dos testes principais evidenciou um ajuste adequado aos modelos de pseudo-primeira ordem e pseudo-segunda ordem. Tanto resultados cinéticos quanto termodinâmicos apontam para o mecanismo de quimissorção do fenol na superfície do carvão ativado.

Embora novas investigações devam ser conduzidas, os resultados deste trabalho indicam que a adsorção de fenol utilizando carvão ativado proveniente do endocarpo do fruto da Macaúba ocorre de forma eficiente, e que esse método possui potencial para ser utilizado na descontaminação de efluentes contendo fenol.

Adicionalmente deve-se citar que o potencial de uso do carvão ativado do endocarpo da Macaúba está ainda em fase de pesquisa tanto nos processos em batelada como no processo por sistemas contínuos, sendo que deverão ser simultaneamente avaliados os seguintes aspectos temporais: resistência mecânica, vida útil, saturação e regeneração por dessorção, disposição após o uso, entre outros.

\section{REFERÊNCIAS}

BRITTO, J. M.; RANGEL, M. C. Processos avançados de oxidação de compostos fenólicos e efluentes industriais. Quim. Nova, v. 31, p. 114-122, 2008.

HO, Y. S.; WASE, D. A. J.; FORSTER, C. F. Kinetic studies of competitive heavy metal adsorption by sphagnum moss peat. Environmental Technology, p. 71-77, 1996.

LANGMUIR, J. The adsorption of gases on planes of glassmica and platinium. Journal American Chemical, v. 40, p. 1361, 1918.

MARSH, H.; REINOSO, F. R. Activated Carbon. Londres: ELSEVIER, 2006.

RODRIGUES, L. A. et al. Phenol removal from aqueous solution by activated carbon produced from avocado kernel seeds. Chemical Engineering Journal, 2011.

SCHNEIDER, E. L. Adsorção de compostos fenólicos sobre carvão ativado. Universidade Estadual do Oeste do Paraná. Toledo - PR, p. 93. 2008.

SPOSITO, G. Soil Science Society America Journal, p. 652, 1980. 